\title{
Comparison of spatial learning in the partially baited radial-arm maze task between commonly used rat strains: Wistar, Spargue-Dawley, Long-Evans, and outcrossed Wistar/Sprague-Dawley
}

\author{
Çiğdem Gökçek-Saraç • Malgorzata Wesierska • \\ Ewa Jakubowska-Doğru
}

Published online: 24 December 2014

(C) Psychonomic Society, Inc. 2014

\begin{abstract}
Strain-related differences in animals' cognitive ability affect the outcomes of experiments and may be responsible for discrepant results obtained by different research groups. Therefore, behavioral phenotyping of laboratory animals belonging to different strains is important. The aim of the present study was to compare the variation in allothetic visuospatial learning in most commonly used laboratory rat strains: inbred Wistar (W) and Sprague-Dawley (SD), outcrossed Wistar/Sprague-Dawley (W/SD), and outbred Long Evans (LE) rats. All rats were trained to the arbitrary performance criterion of $83 \%$ correct responses in the partially baited 12 arm radial maze allowing for simultaneous evaluation of both working and reference memory. In the present study, testing albino versus pigmented and inbred versus outcrossed rats revealed significant strain-dependent differences with the inbred SD rats manifesting lower performance on all learning measures compared to other strains. On the other hand, the outcrossed W/SD rats showed a lower frequency of reference memory errors and faster rate of task acquisition compared to both $\mathrm{LE}$ and $\mathrm{W}$ rats, with $\mathrm{W}$ rats showing a lower frequency of working memory errors compared to other strains. In conclusion, albinism apparently did not reduce the animals' performance in the allothetic visuospatial learning task, while outcrossing improved the spatial learning. A differential effect
\end{abstract}

Ç. Gökçek-Saraç • E. Jakubowska-Doğru ( $₫)$

Department of Biological Sciences, Middle East Technical

University, Ankara, Turkey

e-mail: bioewa@metu.edu.tr

C. Gökçek-Sarac

Department of Environmental Engineering, Faculty of Engineering,

Akdeniz University, Antalya, Turkey

M. Wesierska

Department of Neurobiology, Nencki Institute of Experimental

Biology, 3 Pasteur Street, Warsaw 02093, Poland of strain on the contribution of each error type to the animals' overall performance was observed. The strain-dependent differences were more pronounced between subpopulations of learning-deficient individuals ("poor" learners), and generally the reference memory errors contributed more to the final behavioral output than did the working memory errors.

Keywords Spatial learning - 12-arm radial maze . Reference memory · Working memory $\cdot$ Rat strains

\section{Introduction}

After domestication of the Norway rat (Rattus norvegicus), laboratory rats have become one of the critical animal models for both biomedical and behavioral studies (Whishaw, 2004). Since then, inbred strains and outbred stocks of albino and pigmented rats have been generated and used in neurobiological studies on nervous system function and behavior. A substantial number of these studies have been devoted to the neurobiology of learning and memory, a process that plays a crucial role in shaping animal and human behavior. In the wild, small rodents such as rats and mice live on the ground but also in underground burrows often resembling complicated labyrinths. It is not surprising then that rats and mice are favorable subjects in studies on spatial learning and memory, and that most of the behavioral tasks used in these studies are different types of mazes such as the Olton radial arm maze (Olton \& Samuelson, 1976), the Barnes maze (Barnes, 1979), and the Morris water maze (Morris, Garrud, Rawlins \& O'Keefe, 1982).

Spatial working memory can be defined as short-term storage of a limited amount of spatial information which can be used for other cognitive processes and is available for 
immediate access (Baddeley, 1992). On the other hand, spatial reference memory, a term first coined by Honig (1978) and then used by Olton, Becker \& Handelmann (1979), refers to spatial information which is used over and over again and is usually obtained as a result of repeated training, therefore undergoing consolidation over time and showing higher resistance to interference. It has been well documented that the anatomical and genetic background of these two kinds of memory is not the same (Kandel, 2001; Kandel, 2009). The interplay of environmental and genetic factors (Sousa, Almeida \& Wotjak, 2006; Wahlsten, Metten, Phillips, et al., 2003) and related differences in brain wiring and biochemistry result in individual but also between-strain variations in learning and memory abilities commonly observed in random human or animal populations (Gökçek-Saraç, Karakurt, Adalı \& Jakubowska-Doğru, 2012; Gökçek-Saraç, Adalı \& Jakubowska-Doğru, 2013; Jakubowska-Doğru, Gümüşbaș \& Kara, 2002). Non-cognitive factors such as sensory perception, emotionality, or locomotor activity may also contribute to this variation. It was for instance reported that in some mouse strains (e.g., 129S6 mice) poor Barnes maze performance is related to low exploratory behavior rather than to a spatial memory deficit per se (Holmes, Wrenn, Harris, Thayer \& Crawley, 2002). It has also been postulated that the use of albino rats in visual discrimination tasks might yield data markedly different from the results obtained in similar tasks with pigmented animals (Prusky, Harker, Douglas \& Whishaw, 2002). Strain-related differences in animal performance in cognitive tasks routinely used in laboratory practice cannot be underestimated since such differences affect the outcomes of the experiments where the effects of different manipulations (pharmacologic, surgical, behavioral, or environmental) are studied and compared (Andrews, 1996). They are often a source of discrepant results obtained by different research groups. Therefore, behavioral phenotyping of commonly used laboratory animals such as rats and mice is important. It provides glimpses into the baseline performance of a particular laboratory strain and so has implications for the selection of animal models.

Behavioral differences have been widely studied in mice (Bothe, Bolivar, Vedder \& Geistfeld, 2005; Holmes, Wrenn, Harris, Thayer \& Crawley, 2002; Nguyen, Abel, Kandel \& Bourtchouladze, 2000; Wahlsten, Metten, Phillips, et al., 2003). In rat study literature to date, most available studies focus on strain-dependent differences in sensitivity to pharmacologic agents (Hölscher, 2002; Kacew, 1996; Stöhr, Wermeling, Weiner \& Feldon, 1998), and relatively fewer studies address differences in cognitive functions such as activity-dependent neuroplasticity (Manahan-Vaughan, 2000) and behavioral measures of learning. However, available studies suggest significant differences in performance between strains. For instance, outbred pigmented Long Evans rats were reported to be more successful in autoshaping of a lever press and a two-object discrimination test but worse in a two-island water maze task compared to outbred albino strains, Wistar and Sprague-Dawley (Andrews, Jansen, Linders, Princen \& Broekkamp, 1995). On the other hand, Harker and Whishaw (2002) found wild Long Evans and Fischer-Norway rats superior to inbred Wistar, Fischer 344, Dark-Agouti, and Sprague-Dawley strains in the Morris water maze task (in both place acquisition and matching-to-place performance), suggesting that both inbreeding and albinism but not domestication had adverse effects on cognitive functions. However, in another study, adult pigmented Brown Norway rats demonstrated much poorer performance in an applied variety of tasks including Morris water maze, passive avoidance, delayed non-matching to position, and a shuttle box avoidance task as compared to Wistar, Fischer-344, and Fisher $344 \times$ Brown Norway hybrids (Josef Van Der Staay \& Blokland, 1996). There are, however, relatively few studies comparing the potential strain-related differences in rats using ethologically relevant solid-ground radial arm maze (RAM) in its partially baited version, which enables silmultaneous evaluation of working and reference memory. Considering the independence of these two types of memory (Niewoehner, Single, Hvalby, et al., 2007; Ohno, Yamamoto \& Watanabe, 1993; Woodson, Macintosh, Fleshner \& Diamond, 2003) and their importance in the control of human and animal behavior, the aim of the present study was to analyze individual and strain-dependent variation in both working and reference spatial memory among four common laboratory rat strains including inbred albino Wistar and Sprague-Dawley rats, outcrossed Wistar/Sprague-Dawley rats, and outbred pigmented Long Evans using multiple measures of animal performance in partially baited 12 -arm radial maze.

\section{Materials and methods}

\section{Subjects}

Experiments were carried out on four groups of naïve male rats: 3-month-old inbred albino Wistar $(\mathrm{W})$ rats $(\mathrm{n}=27)$, inbred albino Sprague-Dawley (SD) rats $(\mathrm{n}=29)$, outcrossed Wistar/Spraque-Dawley (W/SD) rats $(\mathrm{n}=33)$, and outbred pigmented Long-Evans (LE) rats $(\mathrm{n}=32)$ (the nomenclature was adapted from Lyon, 1981). All animals were derived from well established colonies. Albino rats were obtained from Gülhane Military Medical Academy (GATA) Animal Facility, Ankara, Turkey, while LE rats came from the Charles River Laboratory, Germany. Prior to and during the experiments, all rats were kept in the Middle East Technical University (METU) animal facility under the same conditions. They were housed in home cages made of transparent plexiglass in groups of three under a constant temperature $\left(21{ }^{\circ} \mathrm{C}\right)$ and a 12/12-hour light/dark cycle with lights on 
between 7 am and $7 \mathrm{pm}$. One week before the experiments, rats were subjected to a food-restricted diet with a daily food portion of about $30 \mathrm{~g}$ of a standard commercial rat chow (Korkutelim Yem Gida San.Tic. A.S.., Turkey) provided to each rat $30 \mathrm{~min}$ after completion of the daily experimental session. Before rats were taken into experiments, their body weight was reduced by $15 \%$ relative to ad libitum weight (Gökçek-Saraç, Adalı \& Jakubowska-Doğru, 2013; GökçekSaraç, Karakurt, Adalı \& Jakubowska-Doğru, 2012; Jakubowska-Doğru, Gümüşbaș \& Kara, 2002). The rats' body weight was recorded daily and kept at a stable level across the experiments. The animal care procedures and all experimental manipulations were pursued in accordance with the METU Ethic Committee Regulations (Protocol No: 2009/01).

\section{Apparatus}

The apparatus was a 12-arm radial maze made of plywood, painted flat grey and elevated $80 \mathrm{~cm}$ above the floor in a room of approximately $12 \mathrm{~m}^{2}$ containing a variety of distal visual cues (a laboratory bench, a sink, shelves, posters), the position of which did not change throughout the experiments. The central platform of the maze was $40 \mathrm{~cm}$ in diameter and arms were $60 \mathrm{~cm}$ long and $9 \mathrm{~cm}$ wide (Gökçek-Saraç, Karakurt, Adalı \& Jakubowska-Doğru, 2012; Gökçek-Saraç, Adalı \& Jakubowska-Doğru, 2013; Jakubowska-Doğru, Gümüşbaş \& Kara, 2002; Wirsching, Beninger, Jhamandas, Boegman \& El-Defrawy, 1984). Each arm was framed by $15-\mathrm{cm}$ high sidewalls made of clear plexiglass which prevented rats from directly crossing from one arm to the other, but allowed them to see the visual cues in the room. A guillotine door was placed at the entrance to each arm. The doors were operated manually and could be moved individually or in concert. A food well ( $2 \mathrm{~cm}$ wide and $2 \mathrm{~cm}$ deep) was located at the end of each arm. To unify the food odor traces throughout the maze, a perforated partition was inserted in all food wells $1 \mathrm{~cm}$ from the bottom, and food pellets (two 2-g chocolate flavored rice puffs; Ülker A.Ș., Turkey), were placed under it. The same food pellets were used as bait during the training. A dim homogenous light was used to illuminate the maze.

\section{Procedure}

\section{Shaping training}

For six consecutive days prior to the experiments all rats were handled daily for 5 min each. At the beginning of RAM experiments, rats were given 5 days of habituation and shaping sessions, in which they were individually allowed to explore the maze for 10 min each day and eat all the food pellets scattered throughout the maze. On each successive day, the number of pellets was reduced and they were placed closer to the ends of the arms. At the end of the shaping training, the rice puffs were placed only in the food wells. Rats were habituated to the movements of guillotine doors on the last day of the shaping. During the shaping training, radial maze was surrounded by non-transparent curtains so the extra-maze cues were invisible to the rats.

\section{RAM training}

During the RAM training, bait was placed only in the food wells of six semi-randomly selected arms, the same for all subjects, ensuring that the spatial relations between baits positions and the distal visuo-spatial cues were the same for all animals (Fig. 1).

One session was given daily. Experiments were run every day between 9 am and $5 \mathrm{pm}$. Rats were trained in groups and always taken into experiments at the same time of day and in the same order. During the daily sessions, rats were individually placed on the central platform facing different directions and allowed to orient themselves. After $5 \mathrm{~s}$ the trial was started by the experimenter concurrently opening all 12 doors and allowing the rat to make its first choice by entering one of the arms. Each time, the rat returned to the central platform after making a choice, the guillotine doors were shut for $5 \mathrm{~s}$, and only then the animal was allowed to make the next choice. This allowed the rats to reorientate and prevented thigmotaxis or chaining behavior. Rats were permitted to choose among the arms until they completed the session by either eating all the pellets or making 12 choices (twice more than the bait in the maze) or $10 \mathrm{~min}$ had passed, whichever came first (Brown \& Giumetti, 2006; Gökçek-Saraç, Karakurt, Adalı \& Jakubowska-Doğru, 2012; Gökçek-Saraç, Adalı \& Jakubowska-Doğru, 2013; Groves, Leslie, Huang et al., 2013; Jakubowska-Doğru, Gümüşbaş \& Kara, 2002; Tarragon, Lopez, Ros-Bernal, et al., 2012; Timberlake \& White, 1990; Wirsching, Beninger, Jhamandas, Boegman \&

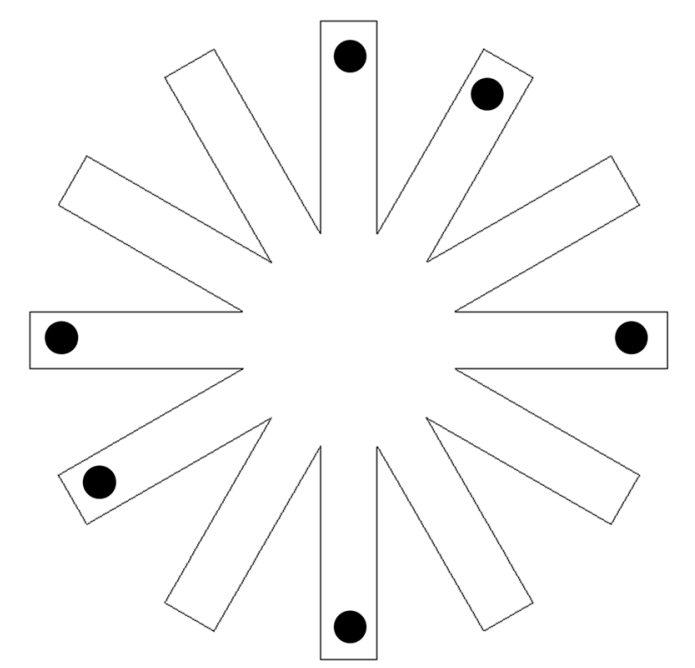

Fig. 1 Schematic representation of the 12-arm radial maze with baited arms marked by black dots 
El-Defrawy, 1984). Entry to the arm was counted only when the rat crossed the midpoint of the alley with its two forepaws. The maze was cleaned of debris and droppings after each rat. All rats were trained according to the criterion of one error or less in the first six choices averaged over three consecutive days of training, which corresponded to a choice accuracy of $83 \%$ or better. A partially baited radial arm maze such as used in the present study allows simultaneous estimation of both reference and working memory errors. To efficiently solve the task, rats must employ a win-stay strategy based on long-term reference memory between foraging sessions, and employ a win-shift strategy based on short-term working memory within a foraging session (Jakubowska-Doğru, Gümüșbaş \& Kara, 2002). In a single experimental session, a successful solution to such a task is not to enter the arms that have never been baited, and not to re-enter the arms that have been depleted of food. Behavioral measures included: (a) number of daily sessions, total number of choices, and total number of errors to the acquisition criterion; (b) total number of entries to unbaited arms (reference memory errors, RMEs); (c) total number of re-entries to either baited or unbaited arms (working memory errors (WMEs)). On the basis of their performance in the radial maze, rats were classified as "good" (total number of choices to the acquisition criterion $\leq$ group mean -3 standard error of mean (SEM)), "poor" (total number of choices to the acquisition criterion $\geq$ group mean $+3 \mathrm{SEM}$ ), and the remaining "intermediate" learners.

\section{Statistical analysis}

Data were expressed as mean \pm SEM. To evaluate the behavioral data, mixed ANOVA (strain $\times$ type of error) followed by one-way ANOVA with either strain or cognitive status ("poor" vs. "good") as independent variable was applied. Fisher's least significant difference (LSD) post hoc test was used for the comparison of simple effects. To evaluate within-group differences between the numbers of working and reference memory errors, Student's $t$-test for paired comparisons was used. To compare the chronological changes in animals' performance in the course of training, two-way repeated measures ANOVA with strain as an independent factor was conducted on percent of correct choices, percent of WMEs, and percent of RMEs out of the first six choices in the consecutive fivesession blocks. Tukey's post hoc tests were used for simple effects comparisons. In this analysis, only the first four fivesession blocks were considered since within the fourth training block all subjects from the W/SD group had reached the acquisition criterion. Prior to analysis, the percent data were converted to arcsine values using Excel ASIN function.

To analyze the correlation between the mean number of total choices with both reference and working memory errors, within and between-strains, the Pearson's correlation test and ANCOVA analysis were applied using the R "cor.test" and "lm" functions, respectively (Team, 2005).

A p-value $\leq 0.05$ was considered as statistically significant. The statistical package SPSS v. 18 was used for other statistical analyses.

\section{Results}

Whole group performance data

The number of sessions, total number of choices, and total number of errors to the acquisition criterion as well as the total numbers of WMEs and RMEs were compared between inbred $\mathrm{W}$ and $\mathrm{SD}$, outbred LE, and outcrossed W/SD rat strains (Fig. 2A, B, C, D).

One-way ANOVA for groups on the total numbers of sessions, choices, and errors to criterion revealed the main effect of strain on all three measures $\left(\mathrm{F}_{(3 ; 120)}=34.243, \mathrm{p} \leq\right.$ $0.001, \mathrm{~F}_{(3 ; 120)}=37.792, \mathrm{p} \leq 0.001$, and $\mathrm{F}_{(3 ; 120)}=38.557$, $\mathrm{p} \leq$ 0.001 , respectively). The post hoc test applied to the group data confirmed the higher overall number of all (sessions, choices, and errors) in SD rats compared to the other strains $(\mathrm{p} \leq 0.001)$ (Fig. 2A, B, C). In outcrossed W/SD rats, the total numbers of sessions to criterion was significantly lower not only compared with SD but also compared with $\mathrm{W}$ and LE rats $(p=0.003$ and $p=0.006$, respectively) (Fig. 2A). A similar pattern of between-strain differences was observed for the total numbers of choices to criterion (Fig. 2B); however, here the difference between $\mathrm{W}$ and $\mathrm{W} / \mathrm{SD}$ rats was not statistically significant $(\mathrm{p}=0.239)$, while the difference between $\mathrm{W}$ and LE rats was only marginally significant $(p=0.07)$. A similar trend was also observed for the total number of errors to criterion (Fig. 2C) with a significantly lower number of errors in $\mathrm{W}$ and $\mathrm{W} / \mathrm{SD}$ strains compared to SD and LE rats, and the difference between $\mathrm{W}$ and W/SD rats was not statistically significant.

In all rat strains, number of emitted RMEs outnumbered that of WMEs (Fig. 2D). Student's $t$-test for paired comparisons yielded the differences between reference and working memory errors as significant in all four strains $(\mathrm{p} \leq 0.001)$.

To evaluate between-strain differences in working and reference memory (Fig. 2D), a mixed ANOVA (Strain (4) $\times$ Error Type (2)) was conducted. The output of the analysis in addition to the main effects of strain and error type $\left(\mathrm{F}_{(3 ; 117)}=\right.$ $38.557, \mathrm{p} \leq 0.001$ and $\mathrm{F}_{(3 ; 117)}=231.951, \mathrm{p} \leq 0.001$, respectively) revealed significant error type $\times$ strain interaction $\left(\mathrm{F}_{(3 ; 117)}=11.264, \mathrm{p} \leq 0.001\right)$. This analysis was followed by one-way ANOVA with strain as the independent variable performed for each type of error type independently. Oneway ANOVA confirmed the effect of strain on both WMEs and RMEs $\left(\mathrm{F}_{(3 ; 120)}=34.823, \mathrm{p} \leq 0.001\right.$ and $\mathrm{F}_{(3 ; 120)}=37.386$, 
A
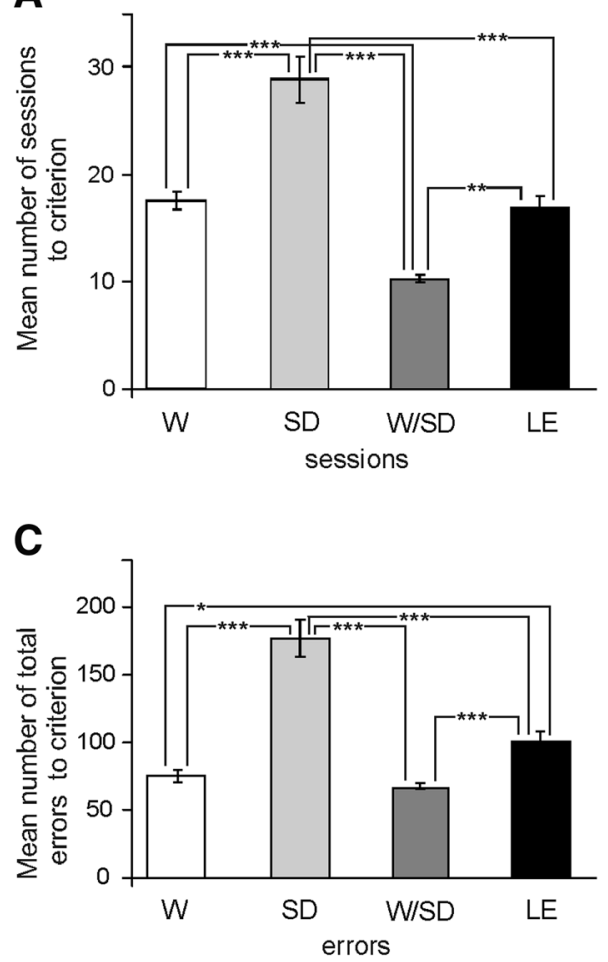

Fig. 2 Mean $( \pm$ SEM) number of sessions $(\mathbf{A})$, choices $(\mathbf{B})$, total errors (C), and mean numbers of working (WME) and reference memory errors (RME) (D) to criterion in W, SD, W/SD, and LE rat strains. Error bars

$\mathrm{p} \leq 0.001$, respectively). The post hoc Tukey test applied to WMEs confirmed a higher emission of these errors in the SD group compared to all three remaining groups $(\mathrm{p} \leq 0.001)$. LE rats emitted more WMEs in comparison to the $\mathrm{W}$ and $\mathrm{W} / \mathrm{SD}$ groups $(\mathrm{p}<0.05)$, whereas the difference between the $\mathrm{W}$ and $\mathrm{W} / \mathrm{SD}$ groups was not significant $(\mathrm{p}=0.491)$. The post hoc test for the occurrence of RMEs showed that the number of these errors was higher in SD rats as compared to other groups $(\mathrm{p} \leq 0.001)$. On the other hand, outcrossed $\mathrm{W} / \mathrm{SD}$ rats made fewer RMEs compared not only to SD and LE rats $(\mathrm{p} \leq 0.001)$ but also compared to $\mathrm{W}$ rats $(\mathrm{p} \leq 0.045)$. The difference in total number of RMEs between LE and $\mathrm{W}$ rats was not statistically significant.
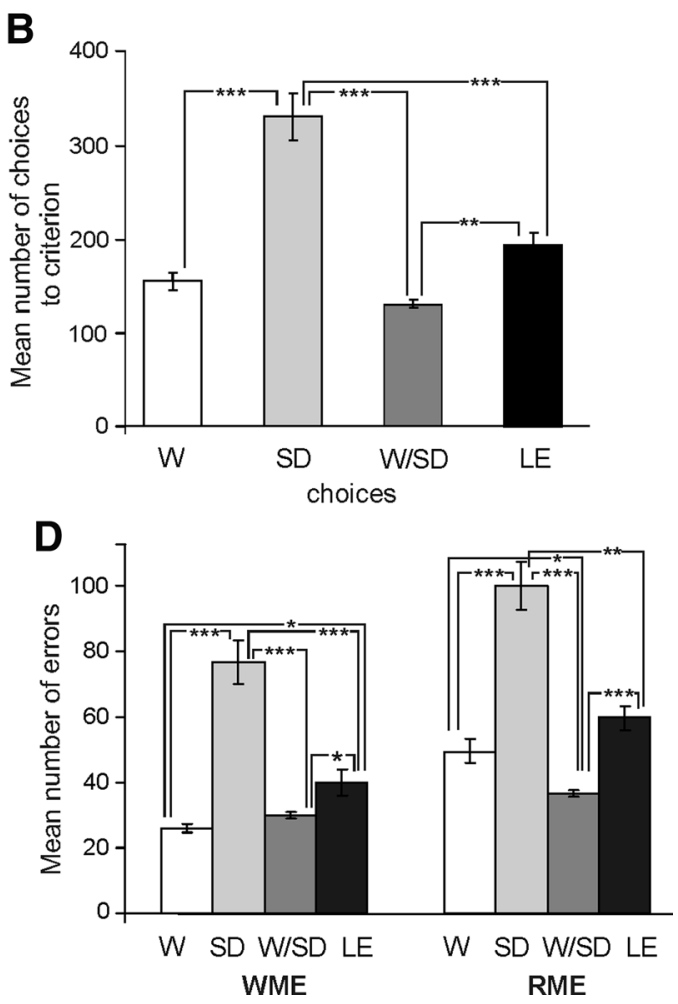

denote \pm SEM. Asterisks denote the level of significance: $* \mathrm{p}<0.05, * * \mathrm{p}$ $<0.01, * * * \mathrm{p}<0.001$

Since the numbers of sessions and choices to criterion differed between groups, we calculated for each rat group the mean number of each type of error per session. The lowest frequency of WMEs/session was estimated for W rats $(1.1 \pm$ $0.0783)$, the second lowest for W/SD rats $(1.8 \pm 0.0850)$, and the highest for LE $(2.3 \pm 0.0955)$ and SD rats $(2.5 \pm 0.1045)$. In contrast to this, the lowest mean frequency of RMEs/session was recorded in W/SD rats $(1.2 \pm 0.0851)$, higher in $\mathrm{W}$ rats $(2.7 \pm 0.1281)$, and highest in SD $(3.3 \pm$ $0.0773)$ and LE rats $(3.4 \pm 0.0641)$.

The strain $\times$ error type interaction revealed through mixed ANOVA may indicate the differential contribution of working and reference memory errors to the groups' overall
A

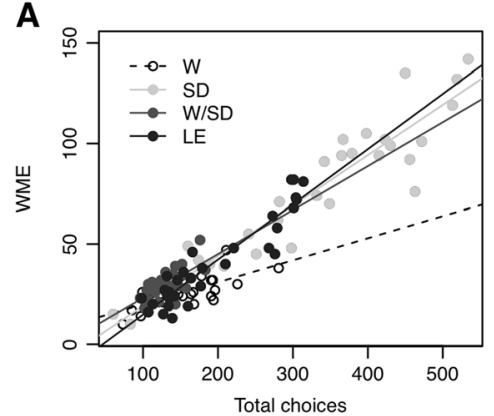

B

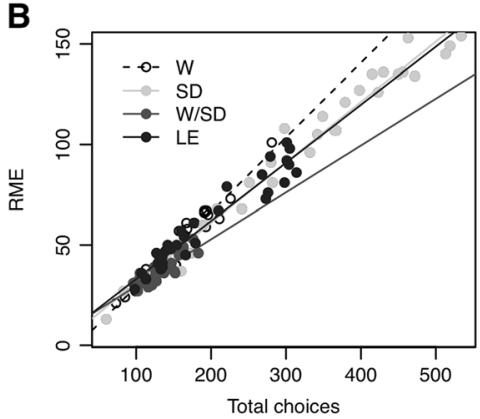

C

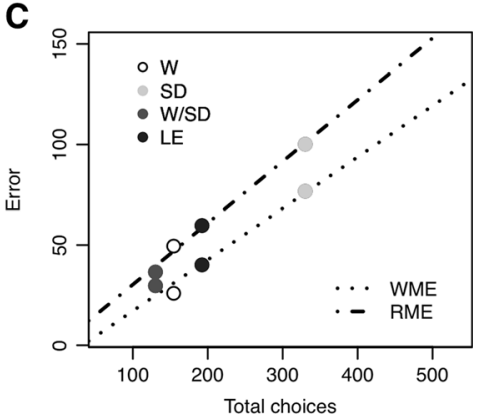

Fig. 3 Regression plots based on individual data $(\mathbf{A}$ and $\mathbf{B})$ and strain means $(\mathbf{C})$ showing the correlation between total number of choices to the task acquisition criterion and total number of WMEs (A), RMEs (B), and total errors (C) 
performance. To evaluate the contribution of each error type to the individual and strain-based variation in the animals' learning skills, Pearson's correlation and ANCOVA analysis with number of choices to criterion as the covariate were performed. Figure 3 presents the regression plots based on individual (A and B) and group (C) data demonstrating a correlation between number of errors (WMEs, RMEs, and total) and the total number of choices to the task acquisition criterion.

Pearson's correlation analysis yielded an overall significant positive correlation between number of choices to criterion and WMEs as well as RMEs $(r=0.98, p=0.015$ and $r=0.99$, $\mathrm{p}=0.004$, respectively). A highly significant positive correlation was also found between these measures in each strain considered independently $(\mathrm{W}: \mathrm{r}=0.72$ and $\mathrm{r}=0.97$, W/SD: $r=0.68$ and $r=0.83$, SD: $r=0.93$ and $r=0.98$, LE: $r=0.93$ and $r=0.96$, for WMEs and RMEs, respectively, $\mathrm{p}<0.0001$ ). Parallel to that, ANCOVA with strain means adjusted for numbers of choices to criterion as covariate yielded significant strain differences for both WME and RMEs ( $\mathrm{p}<10^{-16}$, respectively). Notably, $\mathrm{W}$ rats showed a significant difference for both WME $(\mathrm{p}<0.0001)$ and RMEs $(p<0.0025)$ relative to other strains with a relatively smaller contribution of WMEs to the overall performance, which may be due to a generally lower emission of WMEs compared to other groups.

The learning curves presented in Fig. 4 illustrate changes in the percentage of correct choices as well as working and reference memory errors occurring in the course of training. The learning scores were calculated for the consecutive fivesession training blocks taking into consideration the first six choices in each session. Two-way repeated measures ANOVA with strain as an independent factor applied to the percent of correct choices (transformed to arcsin values) from the first four session blocks (common to all four strains) yielded the main effect of training block, main effect of strain, and training block $\times$ strain interaction highly significant $\left(\mathrm{F}_{(3 ; 351)}=201.132\right.$, $\mathrm{p} \leq 0.0001, \mathrm{~F}_{(3 ; 117)}=19.651, \mathrm{p} \leq 0.0001, \mathrm{~F}_{(9 ; 351)}=21.731, \mathrm{p} \leq$ 0.0001 , respectively). Post hoc comparisons confirmed a significantly slower rate of learning in SD rats compared to all other strains $(p \leq 0.001)$. The rate of learning in outcrossed $\mathrm{W} / \mathrm{SD}$ rats was faster than that in $\mathrm{W}$ and $\mathrm{LE}$ rats $(\mathrm{p} \leq 0.0001)$; however, the difference between $\mathrm{W}$ and LE rats was not statistically significant. Figure $4 \mathrm{C}$ presenting training-dependent changes in the execution of RMEs is a mirror image of Fig. 4A; however, in Fig. 4C, the between-group differences are more pronounced. Analysis of temporal changes in the emission of RMEs also revealed the main effect of training block, main effect of strain, and training block $\times$ strain interaction highly significant $\left(\mathrm{F}_{(3 ; 351)}=218.085, \mathrm{p} \leq 0.0001 ; \mathrm{F}_{(3 ; 117)}=\right.$ $20.900, \mathrm{p} \leq 0.0001$, and $\mathrm{F}_{(9 ; 351)}=23.139, \mathrm{p} \leq 0.0001$, respectively), with post hoc comparisons confirming a significant difference between SD and all three other strains and between $\mathrm{W} / \mathrm{SD}$ and both LE $(\mathrm{p}=0.0001)$ and $\mathrm{W}$ rats $(\mathrm{p}=0.001)$. The difference between $\mathrm{W}$ and LE rats was not statistically significant. The strain $\times$ block interaction indicates different temporal patterns of task acquisition among the strains. A steady but slow decrease in the execution of RMEs paralleled by an increase in percent correct choices was noted in SD rats. Steady but faster changes in both parameters were observed in W/SD and LE rats. In contrast, in W rats, we observed a rapid initial improvement manifested by a significantly lower frequency of RMEs and a higher percent of correct choices in the first five-session block compared to other groups, but then slowing down, resulting in their worse performance in the fourth session block as compared to both W/SD and LE rats. Similar analysis on the emission of WMEs also revealed the main effects of training block, strain, and training block $\times$ strain interaction $\left(\mathrm{F}_{(3 ; 351)}=\right.$ 55.704, $\mathrm{p} \leq 0.0001, \mathrm{~F}_{(3 ; 117)}=14.755, \mathrm{p} \leq 0.0001$, $\mathrm{F}_{(9 ; 351)}=3.671, \mathrm{p} \leq 0.0001$, respectively). Post hoc comparisons revealed significant differences between SD rats
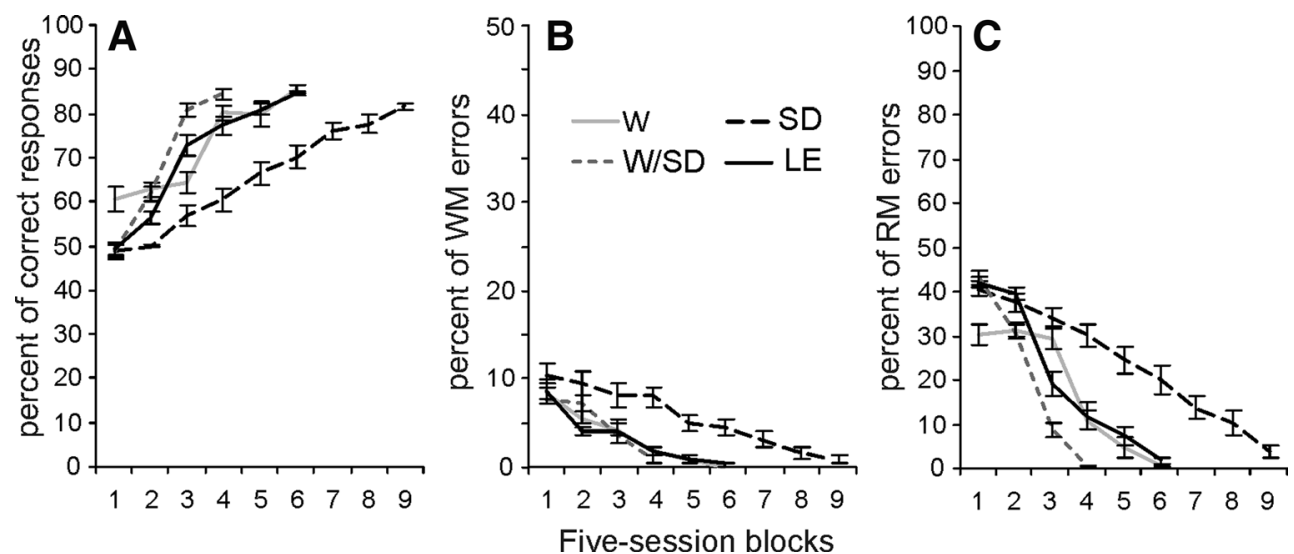

Fig. 4 Mean ( \pm SEM) percent of correct responses (A), mean percent of working memory errors (WMEs) (B), and mean percent of reference memory errors (RMEs) $(\mathbf{C})$ in the first six choices plotted as a

function of five-session blocks in $\mathrm{W}, \mathrm{SD}, \mathrm{W} / \mathrm{SD}$, and $\mathrm{LE}$ rat strains. Error bars denote \pm SEM. Asterisks denote the level of significance: ${ }^{*} \mathrm{p}<0.05,{ }^{*} \mathrm{p}<0.01,{ }^{* * *} \mathrm{p}<0.001$ 
and other strains, while the differences between $\mathrm{W}, \mathrm{W} / \mathrm{SD}$, and LE strains were not statistically significant.

To better analyze the training-dependent changes in animals' performance we applied one-way ANOVAs selectively to the first and the fourth five-session training blocks. Analysis of the data from the first block of sessions revealed a significant strain effect on percentage correct and emission of RMEs but not WMEs $\left(\mathrm{F}_{(3 ; 120)}=12.090, \mathrm{p} \leq 0.0001\right.$, and $\mathrm{F}_{(3 ; 120)}=11.491, \mathrm{p} \leq 0.0001$, respectively). At this early stage of training, post hoc comparison of simple effects confirmed significantly better performance in Wistar rats compared to the other three strains. Analysis of animal performance during the first day of training, however, rendered the strain effect not statistically significant. The same analysis applied to the data from the fourth five-session block revealed a significant main effect of strain on all three measures ( $p \leq 0.0001)$, with SD rats manifesting significantly worse performance compared to the other rats $(\mathrm{p} \leq 0.0001)$. The performance of the outbred W/SD rats as assessed by the percent of RMEs and the percent of correct choices was better compared to all other strains ( $\mathrm{p} \leq$ 0.0001), with the differences between $\mathrm{W}$ and LE rats not statistically significant.

Within- and between- group comparisons of "good" and "poor" learners

The individual rats with a mean number of choices to criterion $\leq$ group mean -3 SEM were classified as "good" learners (W $\mathrm{n}=9, \mathrm{SD} \mathrm{n}=9, \mathrm{~W} / \mathrm{SD} \mathrm{n}=8$, and LE $\mathrm{n}=14$ ), while rats with a mean number of choices to criterion $\geq$ group mean +3 SEM were classified as "poor" learners ( $\mathrm{W} \mathrm{n}=8, \mathrm{SD} n=10, \mathrm{~W} / \mathrm{SD}$ $\mathrm{n}=5$, and LE $\mathrm{n}=10$ ). The remaining animals comprised the intermediate group not taken into consideration in the following analyses. For further analyses animals were divided into groups defined by strain and cognitive status (i.e., W "good", W "poor," etc.). The learning scores for "good" and "poor" learners from each strain are presented in Fig. 5.

One-way ANOVA with group as independent factor applied independently to the numbers of sessions, choices, WMEs, and RMEs to criterion confirmed the main group effect $\left(\mathrm{F}_{(7 ; 72)}=94.277, \mathrm{p} \leq 0.0001, \mathrm{~F}_{(7 ; 72)}=148.795, \mathrm{p} \leq\right.$ $0.0001, \mathrm{~F}_{(7 ; 72)}=62.551, \mathrm{p} \leq 0.0001$, and $\mathrm{F}_{(7 ; 72)}=115.549, \mathrm{p} \leq$ 0.0001 , respectively). In all strains, the differences between "poor" and "good" learners were significant for all four measures. The smallest differences were noted in the outbred $\mathrm{W} / \mathrm{SD}$ rats $(\mathrm{p} \leq 0.05)$ and in the $\mathrm{W}$ group wherein the number of WMEs was only marginally higher $(p=0.063)$ in "poor" compared to "good" learners. All other differences were at $p=$ 0.0001 . According to the results of the post hoc tests (Fischer LSD), SD "good" learners reached the performance criterion significantly more slowly compared to $\mathrm{W}, \mathrm{W} / \mathrm{SD}$, and LE "good" learners as measured by the total number of choices $(p=0.0001, p=0.0001$, and $p=0.006$, respectively) and
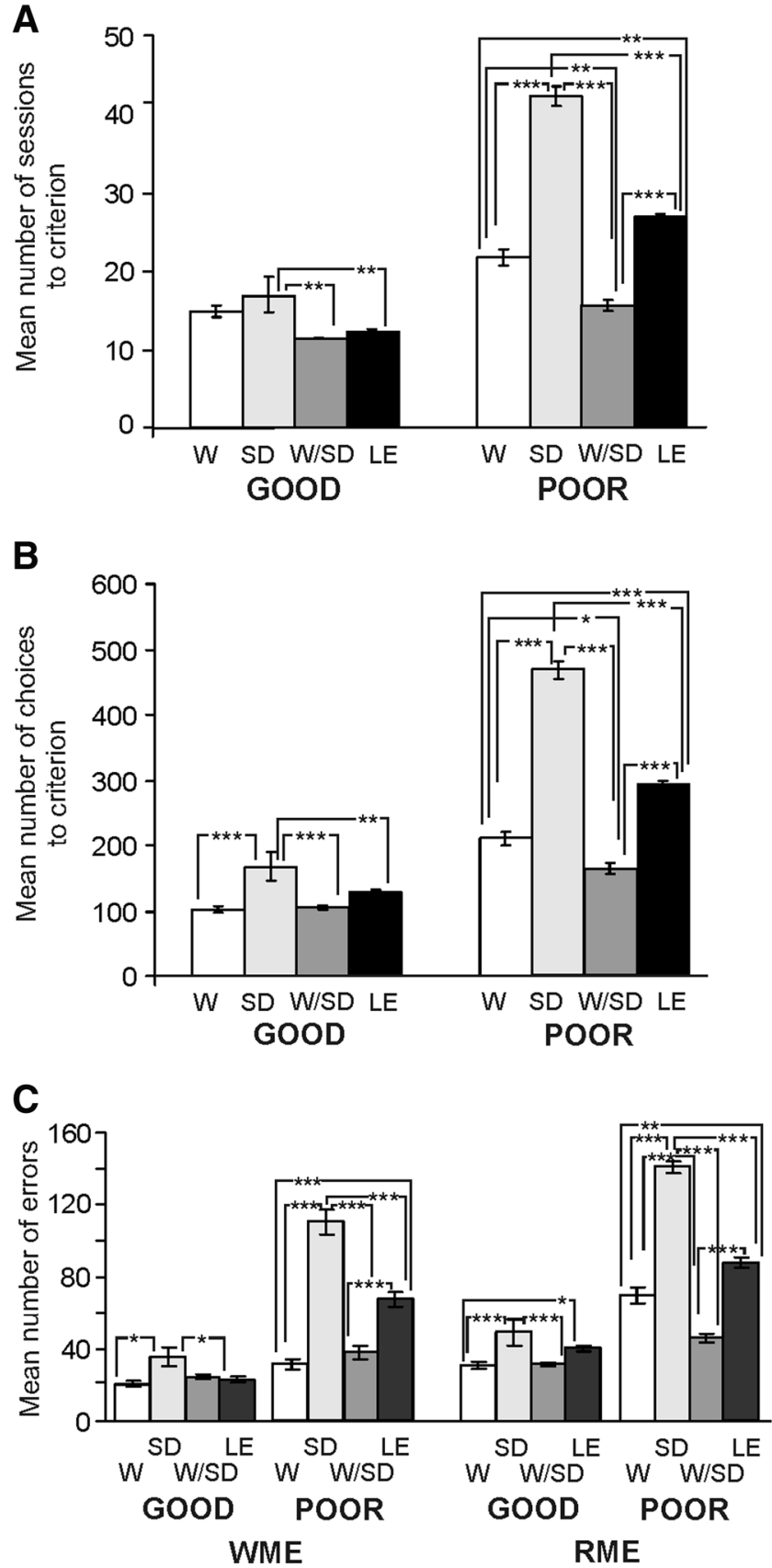

Fig. 5 Mean number ( \pm SEM) of sessions (A), choices (B), working memory errors (WMEs) and reference memory errors (RMEs) (C) to criterion in "good" and "poor" learners from W, SD, W/SD, and LE rat strains. Error bars denote \pm SEM. Asterisks denote the level of significance: $* \mathrm{p}<0.05, * * \mathrm{p}<0.01, * * * \mathrm{p}<0.001$

required significantly more training sessions then W/SD $(\mathrm{p}=$ $0.001)$ and LE $(P=0.002)$, with the difference between SD and W "good" learners not statistically significant. The differences between W, W/SD and LE "good" learners in the rate of learning to criterion were not statistically significant. The same analysis carried out for "good" learners on the numbers of WMEs and RMEs (Fig. 5C) also yielded the main effect of strain highly significant $\left(\mathrm{F}_{(7 ; 72)}=62.551, \mathrm{p} \leq 0.0001\right.$ and 
$\mathrm{F}_{(7 ; 72)}=115.545, \mathrm{p} \leq 0.0001$,respectively). The emission of WMEs was higher in SD "good" learners compared to W, LE, and W/SD "good" learners; however, the difference between SD and W/SD "good" learners was not statistically significant $(p=0.059)$. The differences in the total number of WMEs between W, W/SD and LE "good" learners were also not statistically significant (see Fig. 5B). The execution of RMEs was higher in SD "good" learners compared to all W, W/SD, and LE "good" learners, but the difference between SD and LE "good" learners was not statistically significant $(p=0.070)$. The occurrence of RMEs in LE "good" learners was higher compared to $\mathrm{W}$ "good" learners ( $\mathrm{p}=$ 0.041 ), with no difference between $\mathrm{W}$ and $\mathrm{W} / \mathrm{SD}$ and between W/SD and LE $(p=0.061)$ strains.

Regardless of whether the total number of choices or total number of sessions to criterion was used as a measure, the post hoc comparison of the learning parameters between "poor" learners belonging to different rat strains confirmed slower learning in SD "poor" learners compared to all other strains ( $\mathrm{p}$ $\leq 0.0001$ ). LE "poor" learners were slower in reaching performance criterion than both W and W/SD "poor" learners ( $p$ $\leq 0.0001$ ) and W "poor" learners were slower than W/SD "poor" learners $(p=0.013)$ (Fig. 5A and B). These differences in the rate of reaching task acquisition criterion were paralleled by significant differences in the number of RMEs (Fig. 5C), which was higher in SD "poor" learners than in all other strains ( $\mathrm{p} \leq 0.0001)$, higher in LE "poor" learners compared to $\mathrm{W}$ and W/SD "poor" learners $(\mathrm{p}=0.001$ and $\mathrm{p}=0.0001)$, and higher in $\mathrm{W}$ "poor" learners compared to W/SD "poor" learners ( $\mathrm{p} \leq 0.0001)$. The frequency of WMEs was higher in SD "poor" learners compared to other strains $(\mathrm{p} \leq 0.0001)$, and in LE "poor" learners compared to both, W and W/SD "poor" learners $(\mathrm{p} \leq 0.0001)$ (Fig. 5C).

\section{Discussion}

In the present study, testing albino versus pigmented and inbred versus outcrossed rats representing rat strains most commonly used in behavioral studies on learning and memory revealed significant strain-dependent differences in the rate of reaching an arbitrary performance criterion in the RAM task. In this task where six out of 12 arms were baited, the inbred SD rats manifested poorer performance on all three measures (sessions, choices, and total errors to criterion) compared to the remaining strains while the outcrossed W/SD rats performed better on all measures than the outbred LE rats. The overall performance of inbred $\mathrm{W}$ rats was intermediate between that of W/SD and LE rats.

We used multiple measures to contribute to the discussion which learning parameter - the number of sessions or the cumulative number of choices to the task acquisition criterion - is better as an index of learning in an experimental paradigm such as ours where the number of choices per session differs between the animals. As seen from Figs. 2 and 5 (A and B), the pattern of between-strain differences in these two measures was very similar and the emerging discrepancies rose from between-group differences in the number of choices made by animals during a single session. In our experiments, the difference in the number of sessions to criterion was significantly higher in $\mathrm{W}$ rats compared to W/SD rats, but difference in the number of choices between these two groups was not statistically significant. This discrepancy arises from the higher mean number of choices/ session in W/SD rats $(10.5 \pm 0.351)$ compared to $\mathrm{W}$ rats $(6.6$ \pm 0.755 ), and suggests that a cumulative number of choices is a reliable measure of learning.

The individual and strain-dependent differences in the number of choices made by animals during a single session (10 min in this study), observed especially at the beginning of training, may arise from the differences in animals' locomotor/exploratory activity, and it may potentially have an impact on learning the task. However, our observations argue against it. In this study, no correlation was found between the mean number of choices calculated for the first training day and the first block of five sessions on one hand and the rate of reaching the learning criterion on the other hand. The best learning scores were noted in W/SD rats with the mean number of choices per first training session and the first five-session block equal to $10.5 \pm 0.351$ and $53.03 \pm$ 2.009 , respectively. These values were similar to those recorded for the SD rats having the worst learning scores: $10.5 \pm$ 0.584 and $56.1 \pm 1.309$. In contrast to this, the mean number of choices executed by $\mathrm{W}$ rats considered the second most successful group was $6.6 \pm 0.755$ and $33.37 \pm 0.755$ for the first training session and the first five-session block, respectively. The lack of a direct relationship between the pretraining exploratory activity and space learning in the RAM task was also reported by other authors (Higashida \& Ogawa, 1987).

Individual- and strain-dependent variation in learning skills significantly correlated with the number of both WMEs and RMEs, with RMEs significantly outnumbering the WMEs and thus having a greater contribution to the overall behavioral output. As seen from Fig. 4A and C, changes in the emission of RMEs taking place along the training are a mirror image of the temporal changes in the percent of correct choices. This relatively lower contribution of working memory to solving a six-from-12 radial arm maze task observed in all four rat strains was noted despite the proposal that working memory capacity (in addition to selective attention) is considered to be a "general learning factor," accounting for approximately $40 \%$ of the individual variation in learning skills assessed over multiple learning tasks in mice, another small rodent (Matzel, Wass \& Kolata, 2011).

Considering the independence of working and reference memory, we attempted to separately evaluate the working and 
reference memory performance not only by comparing between groups the total numbers of WMEs and RMEs to criterion but also by conducting a mixed ANOVA (strain $\times$ error Type), Pearson's correlation, and ANCOVA analyses where number of choices to criterion was a covariate of WMEs and RMEs, and finally by separately examining changes in these two error types on chronological basis. Data analyses suggest a differential contribution of each type of error (WME versus RME) to the overall performance of different strains. Between-strain differences were observed in the mean frequency of WMEs and RMEs per session with the lowest mean frequency of WMEs in W and the lowest mean frequency of RMEs in W/SD rats. Also, the mixed ANOVA in addition to the significant main effects of strain and error type revealed significant strain $\times$ error type interaction with the Pearson correlation and ANCOVA analyses, indicating a relatively smaller contribution of WMEs to the overall performance in $\mathrm{W}$ rats as compared to other strains (Fig. 3A). This result gives additional support to the idea of a mechanistic dissociation between working and long-term memory as suggested by some recent studies examining the effects of spatially restricted genetic manipulations confined to the forebrain/hippocampus on animals' performance in the spatial learning tasks allowing for separate recording of working and reference memory errors (Niewoehner, Single, Hvalby, Jensen, Meyer zum Alten Borgloh, Seeburg, Rawlins, Sprengel \& Bannerman, 2007; Sanderson, Good, Skelton, Sprengel, Seeburg, Rawlins \& Bannerman, 2009).

The learning curves presented in Fig. 4 confirm that the learning is a steady incremental process in which an increase in the proportion of correct choices is accompanied by a parallel decrease in both WMEs and RMEs. During the first five-session training block, the choices of SD, W/SD, and LE rats were random and their performance hovered around $50 \%$ correct. Only the performance of $\mathrm{W}$ rats was significantly better, indicating a fast initial task acquisition.

When the performance of rats classified as "good" and "poor" learners was compared between the strains, more pronounced differences were found between the "poor" than the "good" learners. Interestingly, no significant differences were found in the rate of reaching the task acquisition criterion between "good" learners belonging to W, W/SD, and LE strains, while LE "poor" learners were significantly slower in reaching the acquisition criterion and emitted significantly more RMEs than both W/SD and W "poor" learners. On the other hand, W "poor" learner rats were significantly slower in reaching criterion and made significantly more RMEs than W/SD "poor" learners. LE "poor" learners also emitted significantly more WMEs than both W and W/SD "poor" learners. These results suggest that more pronounced between-strain differences in the learning skills occur when comparing individuals with learning scores below the group average while "good" learners from different strains show a ceiling effect.
Reasons for the individual and strain-dependent variation in behavior may differ. Although environmental factors including rearing and maintenance conditions have an impact on animal behavior, whenever differences are found between different lines/strains of laboratory animals coming from well established colonies meeting generally accepted standards of animal care, most probably the nature of such differences is genetic polymorphism, in particular since bi-directional selection for many of these differences seems to be possible (Crusio \& Gerlai, 1999).

According to a naturalistic model of variation of cognitive abilities and the rules of classic genetics, this is an expected result as it is known that outcrossing similar to outbreeding may produce cognitive enhancement due to the masking of deleterious alleles (Fa, Funk \& O'Connell, 2011). The outcrossed W/SD rats had significantly better scores than the inbred SD and LE rats on all three measures, and showed a significantly lower number of RMEs compared to $\mathrm{W}$ rats. The finding that outcrossed W/SD rats were outperforming SD rats but only slightly differed from $\mathrm{W}$ rats may be explained by assuming that the process of genetic differentiation between populations is largely random, and that populations may differ in their accumulation of deleterious recessive mutations and their loss of advantageous alleles through genetic drift. It was postulated that heterosis is maximized in the first generation of interpopulation crosses, and thus the most pronounced effects of heterosis are expected in the first generation following gene flow (Lynch, 1991). On the other hand, several combined behavioral and anatomical studies carried out in small rodents such as rats and mice demonstrated a significant correlation between spatial learning skills and strain-dependent variation in the morphology of the hippocampus (i.e., variation in mossy fibers distribution) (Crusio \& Schwegler, 2005; Prior, Schwegler \& Dücker, 1997). It has also been shown that the architecture of the hippocampus (i.e., mossy fibers terminal fields) has a genetic background and is heritable (Barber, Vaughn, Wimer \& Wimer, 1974; Crusio, Genthner-Grimm \& Schwegler, 2007; Peirce, Chesler, Williams \& Lu, 2003; Vaughn, Matthews, Barber, Wimer \& Wimer, 1977). Parallel to this, profiling of hippocampal gene expression in different mouse strains indicated significant strain-dependent variation in brain protein expression (Gökçek-Saraç, Karakurt, Adalı \& Jakubowska-Doğru, 2012; Pollak, John, Scharl, Leisch, Schneider, Hoeger \& Lubec, 2006).

Interestingly, in our study, albino $\mathrm{W}$ rats known to have worse visual functions compared to pigmented rats including lower visual acuity (Prusky, Harker, Douglas \& Whishaw, 2002) and impaired vision in both bright but also dim light due to light dazzling and lower rod density (Ilia \& Jeffery, 2000) were as good as pigmented LE rats in the rate of learning in this visuospatial task. Harker and Whishaw (2002) in their studies on place learning in the water maze also documented better performance of LE rats compared to 
Fisher-Norway rats, with the latter strain having visual acuity superior to that of LE rats. This observation is in line with findings by Ramos (2000), who reported that rats' performance in the well learned spatial tasks was more impacted by a change in the general shape of the experimental room than by removal of salient extramaze cues. These results may indicate that in small rodents high visual acuity is not necessarily a prerequisite of successful performance in visuospatial tasks where the visual cues are at a distance of $1.5-2 \mathrm{~m}$. However, in contrast to our results, in studies carried out in the 8-arm radial maze, Hooded Lister rats were reported to perform better than Wistar rats in both working memory and in double working and reference memory tasks (ManahanVaughan \& Schwegler, 2011). The superior performance of albino W/SD and on some measures $\mathrm{W}$ rats compared to pigmented LE rats demonstrated in this study is also inconsistent with the results of Harker and Whishaw (2002), who previously reported poorer learning in albino Wistar and Fischer 344 strains compared to pigmented Long Evans and wild rat strains. These discrepancies can be explained by the fact that in addition to visual skills there are several other factors affecting learning. One of them may be the behavioral task used: solid ground maze employing foraging behavior in our experiments and swim-escape response in the MWM in Harker and Whishaw's (2002) studies. In addition, in a dry maze task compared to the water maze, there is a higher probability that some intramaze cues can be used together with the allothetic extramaze room cues to build a cognitive map of the environment which may differentially affect place learning in different strains (Bures, Fenton, Kaminsky, Wesierska \& Zahalka, 1998; Stuchlik, Fenton \& Bures, 2001). Interestingly, in both our and Harker and Whishaw's (2002) studies, regardless of the task used, the SD rats demonstrated the poorest performance in place acquisition.

Taken together, this study demonstrated significant differences in spatial learning between the four most commonly used rat strains, inbred albino Wistar (W) and Sprague-Dawley (SD), outcrossed Wistar/Sprague-Dawley (W/SD) and outbred pigmented Long Evans (LE) rats. These differences were more pronounced between subpopulations of learning-deficient individuals ("poor" learners) compared to "good" learners. In all strains, the reference memory errors had a greater contribution to the final behavioral output than the working memory. A differential effect of strain on the contribution of each type of memory to the animals' overall performance has been noted with $\mathrm{W}$ rats performing relatively less working memory errors compared to other strains. The albinism and thus worse visual functions were shown not to significantly reduce animals' skills for place learning under allothetic navigation cues, while outbreeding significantly improved spatial learning. The results of this and similar studies are important because they help to establish a reference frame for other studies using animal models to investigate mnemonic processes.
Acknowledgments The authors would like to thank Dr. Mehmet Somel for assistance with the statistical evaluation of the data. This work was largely supported by a grant from the Turkish Scientific and Technical Council (TÜBITTAK) to EJD; Project No: 109S133.

\section{References}

Andrews, J. (1996). Possible confounding influence of strain, age and gender on cognitive performance in rats. Cognitive Brain Research, 3(3), 251-267. doi:10.1016/0926-6410(96)00011-0

Andrews, J., Jansen, J., Linders, S., Princen, A., \& Broekkamp, C. (1995). Performance of four different rat strains in the autoshaping, two-object discrimination, and swim maze tests of learning and memory. Physiology \& Behavior, 57(4), 785-790. doi:10.1016/ 0031-9384(94)00336-X

Baddeley, A. (1992). Working memory. Science, 255(5044), 556-559.

Barber, R. P., Vaughn, J. E., Wimer, R. E., \& Wimer, C. C. (1974). Genetically-associated variations in the destribution of dentate granule cell synapses upon the pyramidal cell dendrites in mouse hippocampus. Journal of Comparative Neurology, 156(4), 417-434.

Barnes, C. A. (1979). Memory deficits associated with senescence: A neurophysiological and behavioral study in the rat. Journal of Comparative and Physiological Psychology, 93(1), 74.

Bothe, G. W., Bolivar, V. J., Vedder, M. J., \& Geistfeld, J. G. (2005). Behavioral differences among fourteen inbred mouse strains commonly used as disease models. Comparative Medicine, 55(4), 326-334.

Brown, M. F., \& Giumetti, G. W. (2006). Spatial pattern learning in the radial arm maze. Learn Behav., 34(1), 102-108.

Bures, J., Fenton, A., Kaminsky, Y., Wesierska, M., \& Zahalka, A. (1998). Rodent navigation after dissociation of the allocentric and idiothetic representations of space. Neuropharmacology, 37(4), 689-699. doi:10.1016/S0028-3908(98)00031-8

Crusio, W. E. \& Schwegler, H. (2005). Learning spatial orientation tasks in the radial-maze and structural variation in the hippocampus in inbred mice. Behav Brain Funct, 1(3), 1-11. doi:10.1186/17449081-1-3

Crusio, W., Genthner-Grimm, G., \& Schwegler, H. (2007). A quantitative-genetic analysis of hippocampal variation in the mouse. Journal of Neurogenetics, 21(4), 197-208. doi:10.1080/ 01677060701715827

Crusio, W. E. \& Gerlai, R. T. (Eds.). (1999). Handbook of moleculargenetic techniques for brain and behavior research. Elsevier.

Fa, J. E., Funk, S. M., \& O'Connell, D. (2011). Zoo conservation biology. Cambridge: Cambridge University Press.

Gökçek-Saraç, Ç., Adalı, O., \& Jakubowska-Doğru, E. (2013). Hippocampal levels of ChAT, PKA, phospho-PKA and phosphoCaMKII $\alpha$ but not CaMKII $\alpha$ positively correlate with spatial learning skills in rats. Neuroscience Letters. doi:10.1016/j.neulet.2013. 04.046

Gökçek-Saraç, Ç., Karakurt, S., Adalı, O., \& Jakubowska-Doğru, E. (2012). Correlation between hippocampal levels of neural, epithelial and inducible NOS and spatial learning skills in rats. Behavioural Brain Research. doi:10.1016/j.bbr.2012.08.005

Groves, J. O., Leslie, I., Huang, G. J., McHugh, S. B., Taylor, A., Mott, R., ... Flint, J. (2013). Ablating adult neurogenesis in the rat has no effect on spatial processing: evidence from a novel pharmacogenetic model. PLoS Genetics, 9(9), e1003718. doi:10.1371/journal.pgen. 1003718

Harker, T. K., \& Whishaw, I. Q. (2002). Place and matching-to-place spatial learning affected by rat inbreeding (Dark-Agouti, Fischer 344) and albinism (Wistar, Sprague-Dawley) but not domestication (wild rat vs. Long-Evans, Fischer-Norway). Behavioural Brain Research, 134(1), 467-477. doi:10.1016/S0166-4328(02)00083-9 
Higashida, A., \& Ogawa, N. (1987). Differences in the acquisition process and the effect of scopolamine on radial maze performance in three strains of rats. Pharm. Biochem. Behavi., 27(3), 483-489. doi:10.1016/0091-3057(87)90352-2

Holmes, A., Wrenn, C., Harris, A., Thayer, K., \& Crawley, J. (2002). Behavioral profiles of inbred strains on novel olfactory, spatial and emotional tests for reference memory in mice. Genes, Brain and Behavior, 1(1), 55-69. doi:10.1046/j.1601-1848.2001.00005.x

Hölscher, C. (2002). Different strains of rats show different sensitivity to block of long-term potentiation by nitric oxide synthase inhibitors. European Journal of Pharmacology, 457(2), 99-106. doi:10.1016/ S0014-2999(02)02641-9

Honig, W. K. (1978). Studies of working memory in the pigeon. In S. Hulse, H. Fowler, \& W. K. Honig (Eds), Cognitive processes in animal behavior (pp. 211-248). Hillsdale, NJ: Erlbaum.

Ilia, M., \& Jeffery, G. (2000). Retinal cell addition and rod production depend on early stages of ocular melanin synthesis. Journal of Comparative Neurology, 420(4), 437-444. doi:10.1002/(SICI) 1096-9861(20000515)420:4 < 437::AID-CNE3>3.0.CO;2-1

Jakubowska-Doğru, E., Gümüșbaș, U., \& Kara, F. (2002). Individual variation in the spatial reference and working memory assessed under allothetic and idiothetic orientation cues in rat. Acta Neurobiologiae Experimentalis, 63(1), 17-24.

Josef Van Der Staay, F., \& Blokland, A. (1996). Behavioral differences between outbred Wistar, inbred Fischer 344, brown Norway, and hybrid Fischer $344 \times$ brown Norway rats. Physiology \& Behavior, 60(1), 97-109. doi:10.1016/0031-9384(95)02274-0

Kacew, S. (1996). Invited Review: Role of rat strain in the differential sensitivity to pharmaceutical agents and naturally occuring substances. Journal of Toxicology and Environmental Health Part A, 47(1), 1-30. doi:10.1080/009841096161960-2840

Kandel, E. R. (2001). The molecular biology of memory storage: A dialogue between genes and synapses. Science, 294(5544), 1030 1038. doi: $10.1126 /$ science. 1067020

Kandel, E. R. (2009). The biology of memory: A forty-year perspective. The Journal of Neuroscience, 29(41), 12748-12756. doi:10.1523/ JNEUROSCI. 3958-09.2009

Lynch, M. (1991). The genetic interpretation of inbreeding depression and outbreeding depression. Evolution, 45(3), 622-629.

Lyon, M. F. (1981). Rules for nomenclature of inbred strains. In M. C. Green (Ed.), Genetic variants and strains of the laboratory mouse (1st ed., pp. 368-372). Stuttgart: Gustav Fischer Verlag. ISBN 089574-152-0.

Manahan-Vaughan, D. (2000). Long-term depression in freely moving rats is dependent upon strain variation, induction protocol and behavioral state. Cerebral Cortex, 10(5), 482-487. doi:10.1093/ cercor/10.5.482

Manahan-Vaughan, D., \& Schwegler, H. (2011). Strain-dependent variations in spatial learning and in hippocampal synaptic plasticity in the dentate gyrus of freely behaving rats. Frontiers in Behavioral Neuroscience, 7, 5-7. doi:10.3389/fnbeh.2011.00007

Matzel, L. D., Wass, C., \& Kolata, S. (2011). Individual differences in animal intelligence: Learning, reasoning, selective attention and inter-species conservation of a cognitive trait. International Journal of Comparative Psychology, 24, 36-59.

Morris, R., Garrud, P., Rawlins, J., \& O’Keefe, J. (1982). Place navigation impaired in rats with hippocampal lesions. Nature, 297(5868), 681683.

Nguyen, P. V., Abel, T., Kandel, E. R., \& Bourtchouladze, R. (2000). Strain-dependent differences in LTP and hippocampus-dependent memory in inbred mice. Learning \& Memory, 7(3), 170-179. doi: 10.1101/lm.7.3.170

Niewoehner, B., Single, F. N., Hvalby, Ø., Jensen, V., Meyer zum Alten Borgloh, S., Seeburg, ... Bannerman, D. M. (2007). Impaired spatial working memory but spared spatial reference memory following functional loss of NMDA receptors in the dentate gyrus. European
Journal of Neuroscience, 25: 837-846. doi:10.1111/j.1460-9568. 2007.05312.x

Niewoehner, B., Single, F., Hvalby, Ø., Jensen, V., Meyer zum Alten Borgloh, S., Seeburg, P., ... Bannerman, D. (2007). Impaired spatial working memory but spared spatial reference memory following functional loss of NMDA receptors in the dentate gyrus. European Journal of Neuroscience, 25(3), 837-846. doi:10.1111/j.1460-9568. 2007.05312.x

Ohno, M., Yamamoto, T., \& Watanabe, S. (1993). Blockade of hippocampal nicotinic receptors impairs working memory but not reference memory in rats. Pharmacol. Biochem. Behav. 45, 89-93. doi: 10.1016/0091-3057(93)90091-7

Olton, D. S., \& Samuelson, R. J. (1976). Remembrance of places passed: Spatial memory in rats. Journal of Experimental Psychology: Animal Behavior Processes, 2(2), 97.

Olton, D. S., Becker, J. T., \& Handelmann, G. E. (1979). Hippocampus, space, and memory. Behavioral and Brain Sciences, 2(03), 313-322.

Peirce, J., Chesler, E., Williams, R., \& Lu, L. (2003). Genetic architecture of the mouse hippocampus: Identification of gene loci with selective regional effects. Genes, Brain and Behavior, 2(4), 238-252. doi:10. 1034/j.1601-183X.2003.00030.X

Pollak, D., John, J., Scharl, T., Leisch, F., Schneider, A., Hoeger, H., \& Lubec, G. (2006). Strain-dependent regulation of neurotransmission and actin-remodelling proteins in the mouse hippocampus. Genes, Brain and Behavior, 5(2), 200-204. doi:10.1111/j.1601-183X.2006. 00207. $\mathrm{x}$

Prior, H., Schwegler, H., \& Dücker, G. (1997). Dissociation of spatial reference memory, spatial working memory, and hippocampal mossy fiber distribution in two rat strains differing in emotionality. Behavioural Brain Research, 87(2), 183-194. doi:10.1016/S01664328(97)02282-1

Prusky, G. T., Harker, K. T., Douglas, R. M., \& Whishaw, I. Q. (2002). Variation in visual acuity within pigmented, and between pigmented and albino rat strains. Behavioural Brain Research, 136(2), 339 348. doi:10.1016/S0166-4328(02)00126-2

Ramos, J. M. (2000). Influence of the shape of the experimental room on spatial learning in rats. Physiology \& Behavior, 70(3), 351-357. doi: 10.1016/S0031-9384(00)00266-3

Sanderson, D. J., Good, M. A., Skelton, K., Sprengel, R., Seeburg, P. H., Rawlins, J. N. P., \& Bannerman, D. M. (2009). Enhanced long-term and impaired short-term spatial memory in GluA1 AMPA receptor subunit knockout mice: Evidence for a dual-process memory model. Learning \& Memory, 16(6), 379-386. doi:10.1101/lm.1339109

Sousa, N., Almeida, O., \& Wotjak, C. (2006). A hitchhiker's guide to behavioral analysis in laboratory rodents. Genes, Brain and Behavior, 5(s2), 5-24. doi:10.1111/j.1601-183X.2006.00228.x

Stöhr, T., Wermeling, D. S., Weiner, I., \& Feldon, J. (1998). Rat strain differences in open-field behavior and the locomotor stimulating and rewarding effects of amphetamine. Pharmacology Biochemistry and Behavior, 59(4), 813-818. doi:10.1016/S0091-3057(97)00542-X

Stuchlik, A., Fenton, A., \& Bures, J. (2001). Substratal idiothetic navigation of rats is impaired by removal or devaluation of extramaze and intramaze cues. Proceedings of the National Academy of Sciences, 98(6), 3537-3542. doi:10.1073/pnas.051630498

Tarragon, E. L., Lopez, L., Ros-Bernal, F., Yuste, J. E., Ortiz-Cullera, V., Martin, ... Herrero, M. T. (2012). The Radial Arm Maze (RAM) for the Evaluation of Working and Reference Memory Deficits in the Diurnal Rodent Octodon degus. Journal of Experimental Psychology, 2010(2) 97-116

Team, R. C. (2005). R: A language and environment for statistical computing: ISBN 3-900051-07-0. R Foundation for Statistical Computing. Vienna, Austria, 2013. url: http://www.R-project.org

Timberlake, W., \& White, W. (1990). Winning isn't everything: Rats need only food deprivation and not food reward to efficiently traverse a radial arm maze. Learning and Motivation., 21, 153-163. doi:10. 1016/0023-9690(90)90017-I 
Vaughn, J. E., Matthews, D. A., Barber, R. P., Wimer, C. C., \& Wimer, R. E. (1977). Genetically-associated variations in the development of hippocampal pyramidal neurons may produce differences in mossy fiber connectivity. Journal of Comparative Neurology, 173(1), 41-51.

Wahlsten, D., Metten, P., Phillips, T. J., Boehm, S. L., Burkhart-Kasch, S., Dorow, J., ... Crabbe, J. C. (2003). Different data from different labs: lessons from studies of gene-environment interaction. Journal of Neurobiology, 54(1), 283-311. doi:10.1002/neu.10173

Whishaw, I. Q. (2004). The behavior of the laboratory rat: A handbook with tests. New York: Oxford University Press Inc.
Wirsching, B. A., Beninger, R. J., Jhamandas, K., Boegman, R. J., \& ElDefrawy, S. R. (1984). Differential effects of scopolamine on working and reference memory of rats in the radial maze. Pharmacol Biochem Behav., 20(5), 659-662. doi:10.1016/0091-3057(84) 90180-1

Woodson, J. C., Macintosh, D., Fleshner, M., \& Diamond, D. M. (2003). Emotion-induced amnesia in rats: Working memory-specific impairment, corticosterone-memory correlation, and fear versus arousal effects on memory. Learning \& Memory., 10, 326-336. doi:10. 1101/lm.62903 